\title{
A PROOF OF THE ISOSYSTOLIC INEQUALITY FOR THE KLEIN BOTTLE
}

\author{
TAKASHI SAKAI
}

(Communicated by David G. Ebin)

Dedicated to Professor S. Murakami on his sixtieth birthday

\begin{abstract}
We give a simple proof of the isosystolic inequality for the Klein bottle, which was first obtained by C. Bavard.
\end{abstract}

For a smooth riemannian metric $g$ on the Klein bottle $K$ we consider the metric invariant $\operatorname{Sys} \operatorname{Ar}(g):=\operatorname{Area}(K, g) / \operatorname{sys}^{2}(K, g)$, where sys $(K, g)$ denotes the infimum of the $g$-length of homotopically nontrivial closed curves on $K$. Then C. Bavard obtained the following remarkable inequality.

THEOREM [1]. Sys $\operatorname{Ar}(g) \geq 2 \sqrt{2} / \pi$, where the equality holds for the metric of positive constant curvature with singularities.

Bavard used the result of $\mathrm{Pu}[\mathbf{3}]$ on the geometry of the Möbius band. On the other hand there is also a paper by C. Blatter on the geometry of the Möbius band, and in this note I would like to point out that the above theorem follows directly from the Blatter results.

First, we recall the results of Blatter: Let $\mathbf{S}^{2} \subset \mathbf{R}^{3}$ be the unit sphere and $(\vartheta, \varphi)$ be the Fermi coordinates with respect to the equator $\mathbf{S}^{\mathbf{1}}$. Namely for $m \in$ $\mathbf{S}^{2} \backslash\{$ north and south poles $\}$ we put $\vartheta(m):=\operatorname{dist}_{\mathbf{S}^{2}}\left(m, \mathbf{S}^{\mathbf{1}}\right)$ and $\varphi(m)$ denotes the angular coordinate of the footpoint $p(m)$ of $m$ on $\mathbf{S}^{1}$ with $\operatorname{dist}_{\mathbf{S}^{2}}(m, p(m))=$ $\operatorname{dist}_{\mathbf{S}^{2}}\left(m, \mathbf{S}^{1}\right)$. Now we consider the zone $Z_{\vartheta_{0}}:=\left\{m \in \mathbf{S}^{2} ;|\vartheta(m)|<\vartheta_{0}\right\}(0<$ $\left.\vartheta_{0}<\pi / 2\right)$ and the isometric involution $T$ on $Z_{\vartheta_{0}}$ defined by $T(m):=-m$. The Uniformization Theorem implies that for any Riemannian structure $(M, g)$ on the Möbius band there exist $0<\vartheta_{0}<\pi / 2$ and a positive function $\bar{\rho}$ on $Z_{\vartheta_{0}}$ invariant by $T$, so that $(M, g)$ is isometric to $\left(M_{\vartheta_{0}}, \rho^{2} g_{0}\right):=\left(Z_{\vartheta_{0}}, \bar{\rho}^{2} g_{0}\right) /\{T\}$, where $g_{0}$ denotes the metric of constant curvture 1 . Now let $\Gamma$ be a generator of $\pi_{1}(M) \cong \mathbf{Z}$, which is represented by great circle segments joining $m$ and $T(m)$ and we put $\left.l(g):=\inf _{\text {length }} \gamma ; \gamma \in \Gamma\right\}$. Next let $\Gamma^{*}$ be the family of curves in $M$ defined by $\varphi=$ constant connecting boundary points, which are transversal to the curves $\gamma$ in $\Gamma$ and have the intersection number \pm 1 with $\gamma$. We also put $l^{*}(g):=$ $\inf \left\{\right.$ length $\left.\gamma^{*} ; \gamma^{*} \in \Gamma^{*}\right\}$. Then C. Blatter obtained the following:

Proposition [2]. (1) $\operatorname{Area}(M, g) / l^{2}(g) \geq 2 / \pi \cdot \sin \vartheta_{0}$, where the equality holds iff $\rho$ is equal to a constant.

(2) $\operatorname{Area}(M, g) / l(g) l^{*}(g) \geq \sin \vartheta_{0} / \vartheta_{0}$ if $0<\vartheta_{0}<\Theta$, where $0<\Theta<\pi / 2$ is given by $\tan \Theta=2 \Theta$. The equality holds iff $\rho$ is equal to a constant.

Received by the editors October 20, 1987.

1980 Mathematics Subject Classification (1985 Revision). Primary 53C25.

Key words and phrases. Klein bottle, isosystolic inequality. 
Note that $\Theta>\pi / 3$ for above $\Theta$. Now we shall give our proof of the theorem:

1. Given $(K, g)$, there exists $0<\vartheta_{0}<\pi / 2$ such that $(K, g)$ is the double of $\left(M_{\vartheta_{0}}, g_{1}\right)$ and $\left(M_{\vartheta_{0}}, g_{2}\right)$ with $g_{i}=\rho_{i}^{2} g_{0}(i=1,2)$ and $\rho_{1 \mid \partial M_{\vartheta_{0}}}=\rho_{2 \mid \partial M_{\vartheta_{0}}}$. In fact, $(K, g)$ is conformal to a flat $\left(K, \tilde{g}_{0}\right)$ which is obtained from a rectangle in $\mathbf{R}^{2}$. Then $\left(K, \tilde{g}_{0}\right)$ is the double of the flat Möbius band $\left(M, \tilde{g}_{0}\right)$, which is conformal to $\left(M_{\vartheta_{0}}, g_{0}\right)$ for some $\vartheta_{0}$. Since $\varphi \rightarrow \varphi+\alpha\left(\alpha \in \mathbf{S}^{1}\right)$ are isometries with respect to the metric $\bar{g}_{0}$ on $K$, defined by taking $\rho_{i}=1(i=1,2)$, we may assume that $\rho_{i}$ depend only on $\vartheta$ by the standard argument averaging $\rho_{i}$ by the action of $\mathbf{S}^{1}$ (see $[2, \mathbf{3}])$. Then we have easily

$$
\operatorname{Area}(K, g)=\operatorname{Area}\left(M_{\vartheta_{0}}, g_{1}\right)+\operatorname{Area}\left(M_{\vartheta_{0}}, g_{2}\right)
$$

and

$$
\operatorname{sys}(K, g) \leq \min \left\{l\left(g_{1}\right), l\left(g_{2}\right), l^{*}\left(g_{1}\right)+l^{*}\left(g_{2}\right)\right\} .
$$

2. (Case when $\pi / 2>\vartheta_{0} \geq \pi / 4$ ). We have from (1) of the Proposition

$$
\begin{gathered}
\operatorname{Sys} \operatorname{Ar}(K, g) \geq \operatorname{Area}\left(M_{\vartheta_{0}}, g_{1}\right) / l^{2}\left(g_{1}\right)+\operatorname{Area}\left(M_{\vartheta_{0}}, g_{2}\right) / l^{2}\left(g_{2}\right) \\
\geq 2 \cdot 2 / \pi \cdot \sin \vartheta_{0} \geq 4 / \pi \cdot \sin \pi / 4=2 \sqrt{2} / \pi,
\end{gathered}
$$

where the equality holds iff $\vartheta_{0}=\pi / 4$ and $\rho_{1}=\rho_{2}=$ constant.

3. (Case when $0<\vartheta_{0} \leq \pi / 4$ ). We have from (2) of the Proposition,

$$
\begin{aligned}
\operatorname{Sys} & \operatorname{Ar}(K, g) \geq\left\{\operatorname{Area}\left(M_{\vartheta_{0}}, g_{1}\right)+\operatorname{Area}\left(M_{\vartheta_{0}}, g_{2}\right)\right\} /\left\{\operatorname{sys}(K, g)\left(l^{*}\left(g_{1}\right)+l^{*}\left(g_{2}\right)\right\}\right. \\
& \geq \min \left\{\operatorname{Area}\left(M_{\vartheta_{0}}, g_{1}\right) / l\left(g_{1}\right) l^{*}\left(g_{1}\right), \operatorname{Area}\left(M_{\vartheta_{0}}, g_{2}\right) / l\left(g_{2}\right) l^{*}\left(g_{2}\right)\right\} \\
& \geq \sin \vartheta_{0} / \vartheta_{0} \geq(\sin \pi / 4) /(\pi / 4)=2 \sqrt{2} / \pi,
\end{aligned}
$$

because $\vartheta \rightarrow \sin \vartheta / \vartheta$ is strictly monotone decreasing on $(0, \pi / 2]$. The equality holds iff $\vartheta_{0}=\pi / 4$ and $\rho_{1}=\rho_{2}=$ constant.

4. It is easy to see that for the Riemannian metric $\left(K, \bar{g}_{0}\right)$ obtained in the above by the condition $\vartheta_{0}=\pi / 4$ and $\rho_{1}=\rho_{2}=$ constant, $\operatorname{Sys} \operatorname{Ar}\left(K, \bar{g}_{0}\right)$ is in fact equal to $2 \sqrt{2} / \pi$. Note that $\bar{g}_{0}$ is a continuous Riemannian metric and smooth except at $\partial M_{\vartheta_{0}}$.

\section{REFERENCES}

1. C. Bavard, Inégalité isosystolique pour la bouteille de Klein, Math. Ann. 274 (1986), 439-441.

2. C. Blatter, Zur Riemannschen Geometrie in Grossen auf der Möbius band, Compositio Math. 15 (1961), 88-107.

3. P. $\mathrm{Pu}$, Some inequalities in certain non-orientable Riemannian manifolds, Pacific J. Math. 2 (1952), 55-72. 